\title{
Comparison of Combined Tofogliflozin and Glargine, Tofogliflozin Added to Insulin, and Insulin Dose-Increase Therapy in Uncontrolled Type 2 Diabetes
}

\author{
Katsunori Suzukia, c, Yurie Mitsuma ${ }^{a}$, Takaaki Sato ${ }^{a}$, Takumi Anraku ${ }^{a}$, Mariko Hatta ${ }^{b}$
}

\begin{abstract}
Background: Some patients with type 2 diabetes mellitus (T2DM) on insulin have poor glycemic control and require add-on therapy to reach target glucose values. Increased insulin doses or the addition of an oral antidiabetic drug (OAD) may improve glycemic control, but many patients fail to achieve target values. The aim of this study was to compare the treatment efficacy and safety of three different therapies in such patients.
\end{abstract}

Methods: T2DM outpatients with poor glycemic control (HbA1c $\geq$ $7.0 \%$ ) despite insulin therapy (including patients on OADs other than a sodium-glucose cotransporter 2 (SGLT2) inhibitor) were included. The patients had a body mass index (BMI) of $\geq 22 \mathrm{~kg} / \mathrm{m}^{2}$ and an estimated glomerular filtration rate (eGFR) of $\geq 45 \mathrm{~mL} / \mathrm{min} / 1.73 \mathrm{~m}^{2}$, did not have depletion of endogenous insulin, and had stable glucose levels for 3 months before study entry on insulin therapy. Treatment was continued for 24 weeks with insulin dose-increase therapy, tofogliflozin add-on therapy, or a combination of insulin glargine + tofogliflozin. The primary endpoints were $\mathrm{HbAlc}$, weight, and total insulin dose. Secondary endpoints included fasting plasma glucose (FPG), blood pressure, lipid profiles, and incidence of adverse events

Results: At baseline, the participants' median age was 59.0 years, mean BMI was $28.7 \mathrm{~kg} / \mathrm{m}^{2}$, mean eGFR was $89.2 \mathrm{~mL} / \mathrm{min} / 1.73 \mathrm{~m}^{2}$, mean $\mathrm{HbA} 1 \mathrm{c}$ was $8.7 \%$, and mean FPG was $174.1 \mathrm{mg} / \mathrm{dL}$. The mean duration of insulin therapy was approximately 7 years. The mean daily insulin dose was approximately $40 \mathrm{U}$ in the three groups. Overall, $85 \%$ received other background OADs in addition to insulin. Over the 24-week period, HbAlc in the insulin group decreased slightly initially and then plateaued; daily total insulin dose and weight increased, and blood pressure increased slightly. In the insulin + tofogliflozin group and the glargine + tofogliflozin group, $\mathrm{HbA} 1 \mathrm{c}$ decreased greatly initially, and this continued over the 24-week period, with $\mathrm{HbA} 1 \mathrm{c}$

Manuscript accepted for publication September 21, 2016

aDivision of Endocrinology and Metabolism, Saiseikai Niigata Daini Hospital, Niigata, Japan

bDepartment of Nutrition, Saiseikai Niigata Daini Hospital, Niigata, Japan ${ }^{c}$ Corresponding Author: Katsunori Suzuki, Division of Endocrinology and Metabolism, Saiseikai Niigata Daini Hospital, 280-7 Teraji, Nishi-ku, Japan. Email: katsu-s@ngt.saiseikai.or.jp

doi: http://dx.doi.org/10.14740/jocmr2741w decreases of $-1.0 \%$ and $-0.8 \%$, respectively; total daily insulin dose ( -2.6 and $-12.7 \mathrm{U}$, respectively) and weight $(-2.9$ and $-3.4 \mathrm{~kg}$, respectively) decreased, and blood pressure decreased slightly. Tofogliflozin therapy was well tolerated.

Conclusions: Tofogliflozin may offer a new option for patients whose T2DM remains inadequately controlled on insulin therapy with or without additional oral glucose-lowering agents.

Keywords: Glycemic control; SGLT2 inhibitor; Tofogliflozin; Insulin; Insulin glargine; Type 2 diabetes mellitus

\section{Introduction}

Physicians in clinical practice often encounter patients with type 2 diabetes mellitus (T2DM) on insulin who have poor glycemic control and require add-on therapy to reach target glucose values. Increased insulin doses or the addition of an oral antidiabetic drug (OAD) may improve glycemic control, but many patients still do not achieve target values [1-3]. Insulin therapy using basal and mixed insulin may effectively reduce fasting plasma glucose (FPG), but appropriate control of acute rises in postprandial glucose levels is often more difficult [4-6]. Moreover, even if insulin dose adjustments can improve glycemic control, insulin doses often cannot be increased because of a risk of hypoglycemia and weight gain [7, 8]. Therefore, the ideal treatment of T2DM patients aims at glycemic control without hypoglycemia, without weight gain, and with minimal glycemic excursions.

Interest has focused on the kidney as a potential new therapeutic target in patients with T2DM because the kidney filters and reabsorbs approximately $180 \mathrm{~g}$ of glucose per day [9], and, in T2DM, renal glucose reabsorption is maladaptively increased $[10,11]$. The sodium-glucose cotransporter 2 (SGLT2) is a low-affinity, high-capacity transporter located in the S1 segment of the proximal convoluted tubule of the nephron that mediates the majority of renal glucose reabsorption from the glomerular filtrate.

Tofogliflozin, a competitive and highly selective inhibitor of SGLT2, reduced renal glucose reabsorption, dose-dependently increased urinary glucose excretion [12], and reduced hyperglycemia in patients with T2DM $[13,14]$. Therefore, 
among the currently available SGLT2 inhibitors, tofogliflozin excels for reducing postprandial glucose [15]. Because tofogliflozin acts independently of insulin secretion or action, it may provide additional glycemic control when used with insulin in patients with advanced $\beta$-cell failure [16]. Moreover, energy loss and osmotic diuresis secondary to increased urinary glucose excretion and natriuresis may counter insulin-related weight gain and fluid retention, respectively.

A greater number of insulin injections in T2DM patients increase treatment burden and may be a reason for reduced patient adherence to diabetes treatment $[17,18]$. Changes to improve glycemic control such as switching from one or two to more frequent insulin injections daily, increasing from one type to two different types of insulin, and in some cases, increasing insulin injections to 4 - 5 times daily are often difficult to achieve in outpatient settings. Therefore, if patients with poor glycemic control despite frequent insulin treatment can safely be switched to basal supported oral therapy (BOT) using basal insulin and an SGLT2 inhibitor, this can improve glycemia in outpatient care without reducing patient quality of life (QOL).

Therefore, in this study, patients with poor glycemic control despite insulin therapy were divided into three groups to receive treatment with one of insulin dose-increase therapy, tofogliflozin add-on therapy, or a combination of insulin glargine + tofogliflozin, and treatment efficacy and safety were compared.

\section{Materials and Methods}

This study included T2DM patients with poor glycemic control $(\mathrm{HbA} 1 \mathrm{c} \geq 7.0 \%)$ despite insulin therapy (including patients on OADs other than an SGLT2 inhibitor) treated in our outpatient clinic between June 2014 and June 2015. The included patients had a body mass index (BMI) of $\geq 22 \mathrm{~kg} / \mathrm{m}^{2}$ and an estimated glomerular filtration rate (eGFR) of $\geq 45 \mathrm{~mL} / \mathrm{min} / 1.73 \mathrm{~m}^{2}$, did not have depletion of endogenous insulin, and had stable glucose levels for 3 months before study entry on insulin therapy (dose $\geq 10$ units/day for $\geq 6$ months). The nature of the study was explained, and informed consent was obtained from all participants.

The patients were randomly assigned in a 1:1:1 ratio into three groups for treatment with insulin dose-increase therapy (insulin group), tofogliflozin add-on therapy (insulin + tofogliflozin group), or a combination of insulin glargine + tofogliflozin (glargine + tofogliflozin group). Treatment was continued for 24 weeks according to the protocol described below. The primary endpoints in this prospective study were HbA1c, weight, and total insulin dose. Secondary endpoints included FPG, blood pressure, lipid profiles, and the incidence of adverse events.

In the insulin group, insulin doses were adjusted according to an "insulin dose algorithm" (Table 1) to achieve a target FPG of $110-130 \mathrm{mg} / \mathrm{dL}$. Basal and mixed insulin doses were based on average pre-breakfast glucose levels for 3 days before an outpatient visit. These doses were titrated according to the dose adjustment algorithm (Table 1). This algorithm was used only if there were no episodes of severe hypoglycemia. Patients on basal-bolus insulin therapy were asked to first adjust their basal insulin dose before considering a change in their bolus
Table 1. Insulin Dose Algorithm

\begin{tabular}{|ll}
\hline $\begin{array}{l}\text { Mean pre-breakfast glucose } \\
\text { value and/or mean pre-dinner } \\
\text { glucose value (mg/dL) }\end{array}$ & $\begin{array}{l}\text { Adjustment of basal insulin } \\
\text { or mixed insulin dose (units) }\end{array}$ \\
\hline$\leq 56$ & -4 \\
\hline $56-80$ & -2 \\
\hline $81-110$ & No adjustment \\
\hline $111-126$ & 1 \\
\hline $127-144$ & 2 \\
\hline $145-162$ & 3 \\
$\geq 163$ & $\geq 4$ \\
\hline
\end{tabular}

Bolus insulin dose adjusted at discretion of treating physician only if no episodes of severe hypoglycemia. Outpatient visits every 2 weeks for first few months in the insulin + tofogliflozin group and glargine + tofogliflozin group.

insulin dose (excluding when the treating physician deemed it absolutely necessary that the bolus insulin dose first be adjusted). Bolus insulin doses were adjusted at the discretion of the treating physician. Priority over this algorithm was given to the clinical judgement of the treating physician to avoid adversely affecting the patients' safety. In addition, if measured glucose levels were low without any specific cause, the basal or mixed insulin doses were decreased as shown in Table 1.

In the insulin + tofogliflozin group, the insulin dose remained fixed, and tofogliflozin $20 \mathrm{mg}$ once daily after breakfast was given as add-on therapy. In the glargine + tofogliflozin group, the starting dose of glargine (once daily in the morning) was 0.7 units $\times$ the total units of insulin per day of all insulin preparations used up to that time. Tofogliflozin $20 \mathrm{mg}$ once daily after breakfast was given as add-on therapy.

In all three groups, OADs that were already being used were continued during the study period. Patients used with other drugs that might affect glucose metabolism were excluded during the study.

Patients were instructed to follow a stable diet and exercise regimen after enrolment. The protocol was approved by institutional review boards and/or independent ethics committees (E14-06), and all participants gave their written, informed consent. The study was carried out in accordance with the principles of the Declaration of Helsinki (2004 version) and the requirements of Good Clinical Practice.

The data were expressed as mean (standard deviation (SD)) values. Statistical analysis included the paired $t$-test and the Mann-Whitney test using GraphPad Prism Version 6.07. The level of significance (two-sided) was $5 \%$.

\section{Results}

\section{Patients}

At entry into the study, the median age of the participants was 59.0 years, the mean BMI was $28.7 \mathrm{~kg} / \mathrm{m}^{2}$, and the mean eGFR was $89.2 \mathrm{~mL} / \mathrm{min} / 1.73 \mathrm{~m}^{2}$. The mean duration of insulin ther- 
Table 2. Demographic and Baseline Patient Characteristics

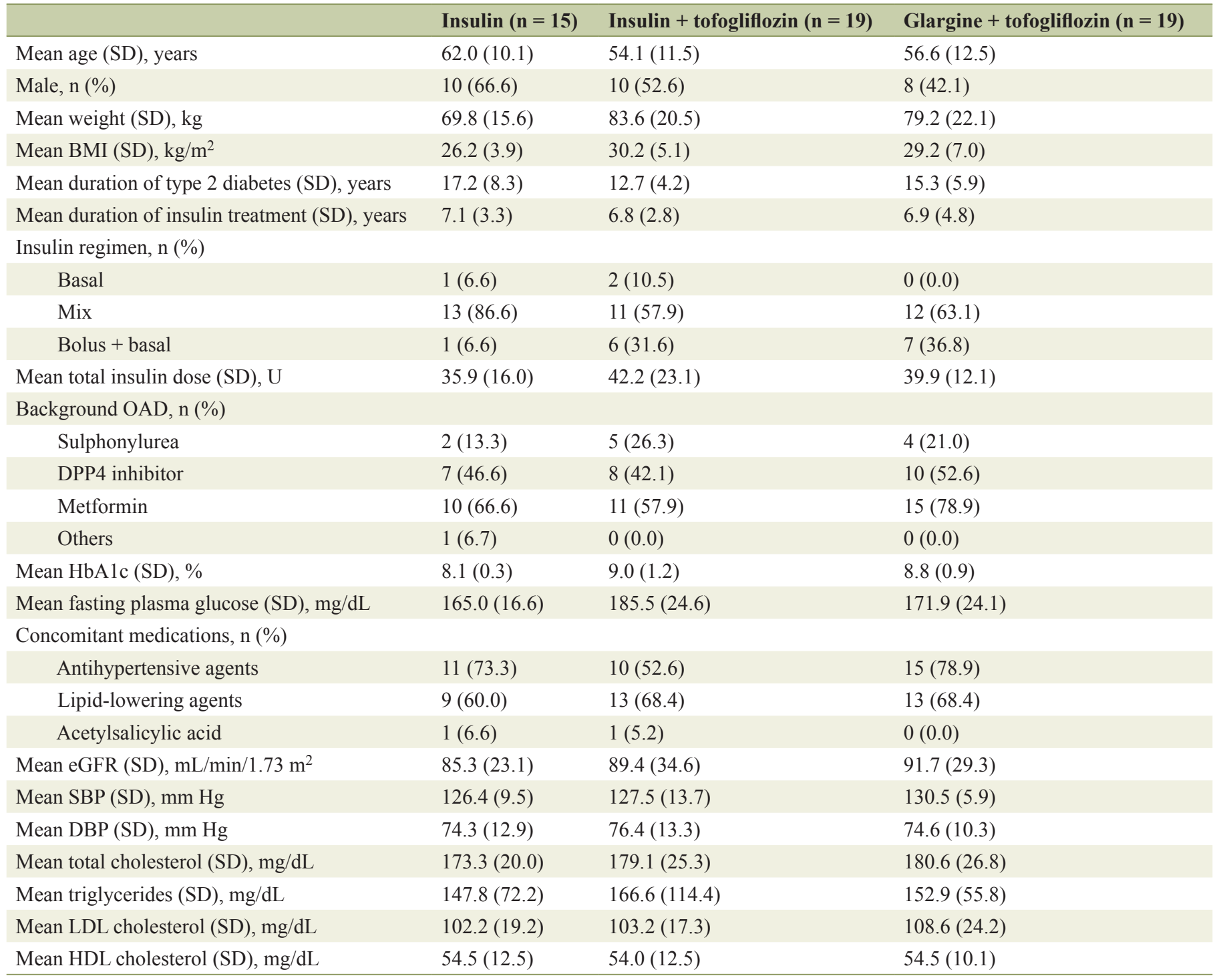

$\mathrm{n}$ : number of patients in the full analysis set. BMI: body mass index; OAD: oral antidiabetic drug; SD: standard deviation; SBP: systolic blood pressure; DBP: diastolic blood pressure. All characteristics were balanced across the randomized groups at baseline.

apy was approximately 7 years, around half of the time since the diagnosis of T2DM (14.9 years). The mean daily insulin dose was approximately $40 \mathrm{U}$ in the three groups. Overall, $85 \%$ received other background OADs in addition to insulin (principally metformin), the mean baseline $\mathrm{HbA1c}$ was $8.7 \%$, and the mean baseline FPG was $174.1 \mathrm{mg} / \mathrm{dL}$. Table 2 summarizes the baseline characteristics of the patients in the three groups. Most patients in each group were using mixed insulin, followed next by patients using bolus + basal insulin. All characteristics were balanced across the randomized groups at baseline.

\section{Change in HbA1c and FPG}

Mean HbA1c levels (95\% confidence interval (CI)) decreased significantly from baseline to after 4 weeks in all three groups. The mean change in HbA1c from baseline to 24 weeks was $-0.3 \%$ in the insulin group ( $95 \% \mathrm{CI}:-0.60$ to 0.02$),-1.0 \%$ in the insulin + tofogliflozin group (95\% CI: -1.49 to -0.59 ), and $-0.8 \%$ in the glargine + tofogliflozin group (95\% CI: -1.28 to -0.22) (Fig. 1 and Table 3). Regarding between-group differences, compared to the insulin group, the mean reduction in HbAlc was significantly greater in the insulin + tofogliflozin group (-0.65\%, $\mathrm{P}=0.0052)$ (Fig. 1).

The mean changes (significant decreases) in FPG from baseline to 24 weeks were $-10.9 \mathrm{mg} / \mathrm{dL}$ in the insulin group (95\% CI: -20.9 to $-0.87 \mathrm{mg} / \mathrm{dL}),-61.3 \mathrm{mg} / \mathrm{dL}$ in the insulin + tofogliflozin group (95\% CI: -81.9 to $-39.8 \mathrm{mg} / \mathrm{dL})$, and -33.1 $\mathrm{mg} / \mathrm{dL}$ in the glargine + tofogliflozin group (95\% CI: -46.4 to $-19.7 \mathrm{mg} / \mathrm{dL}$ ) (Fig. 2 and Table 3). 


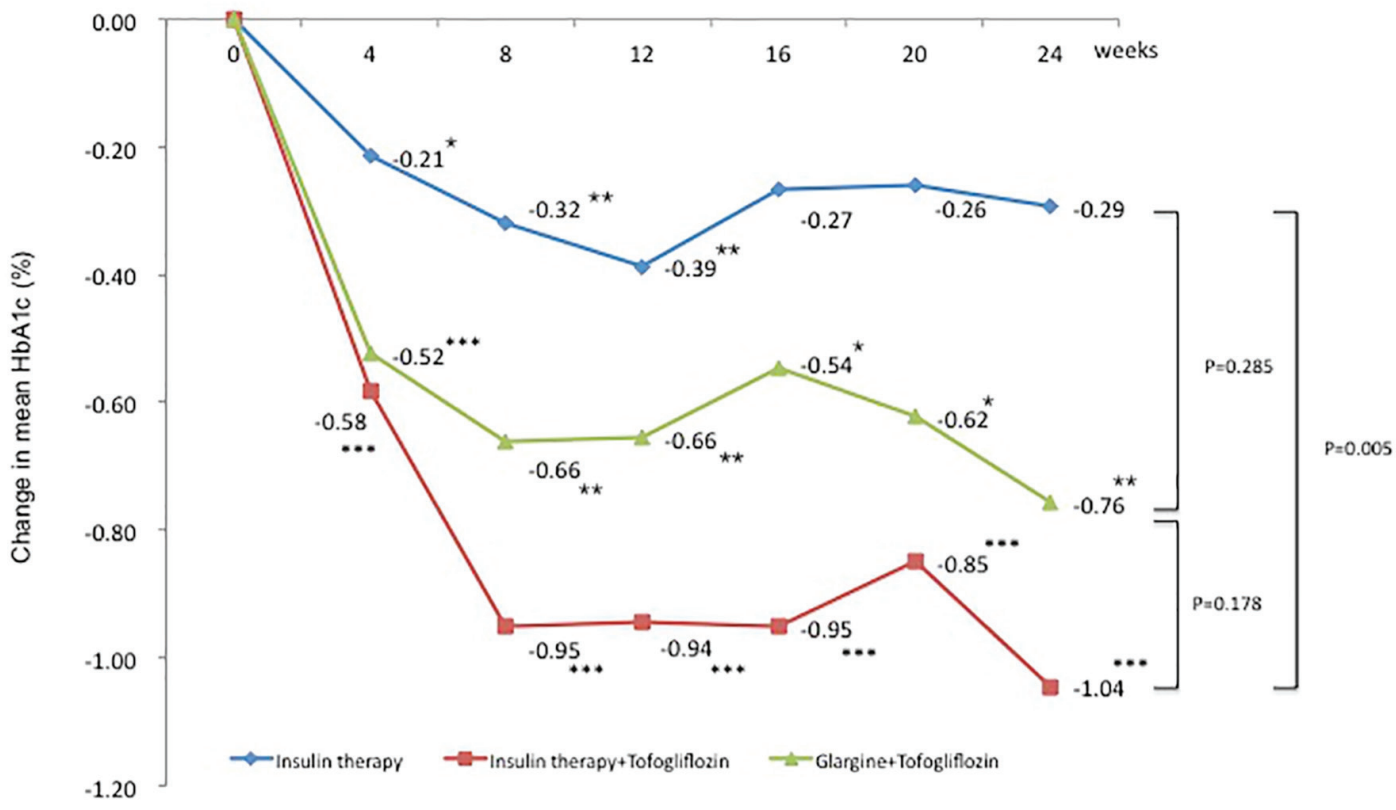

Figure 1. Adjusted mean changes over 24 weeks in $\mathrm{HbA} 1 \mathrm{c}(\%)$. Data are adjusted mean \pm standard deviation (SD) at baseline and adjusted mean \pm SD on treatment. ${ }^{*} P<0.05$ vs. baseline; ${ }^{* \star} P<0.01 \mathrm{vs}$. baseline; ${ }^{* \star \star} P<0.001$ vs. baseline by paired $t$-test. Above $\mathrm{P}$ values were derived using Mann-Whitney analysis to examine between-group differences.

\section{Change in insulin dose}

The change in mean total daily insulin dose from baseline was $5.1 \mathrm{U}$ in the insulin group (95\% CI: 3.10 to $7.02 \mathrm{U})$, a significant increase of $14.2 \%$. In contrast, the changes in mean total daily insulin dose from baseline were $-2.6 \mathrm{U}$ in the insulin + tofogliflozin group (95\% CI: -5.57 to $0.45 \mathrm{U}$ ) and $-12.7 \mathrm{U}$ in the glargine + tofogliflozin group $(95 \% \mathrm{CI}:-15.2$ to -10.2 $\mathrm{U})$. These were significant decreases of $-6.2 \%$ and $-34.8 \%$, respectively (Fig. 3 and Table 3). Regarding between-group differences, compared to the insulin group, the mean reduction in insulin dose was significantly greater in the insulin + tofogliflozin group $(-0.4 \mathrm{U}, \mathrm{P}<0.0001)$ and in the glargine + tofogliflozin group (-17 U, P $<0.0001)$ (Fig. 3).

\section{Change in weight}

The change in mean weight from baseline was $0.6 \mathrm{~kg}$ in the insulin group (95\% CI: -0.28 to $1.39 \mathrm{~kg}$ ), which was a slight increase. In contrast, the changes in mean weight from baseline were $-2.9 \mathrm{~kg}$ in the insulin + tofogliflozin group (95\% CI: -4.46 to $-1.27 \mathrm{~kg}$ ) and $-3.4 \mathrm{~kg}$ in the glargine + tofogliflozin group (95\% CI: -4.87 to $-1.91 \mathrm{~kg})$. These were significant decreases (Fig. 4 and Table 3 ). Regarding between-group differences, compared to the insulin group, the mean reduction in weight was significantly greater in the insulin + tofogliflozin group $(-2.95 \mathrm{~kg}, \mathrm{P}=0.0001)$ and in the glargine + tofogliflozin group $(-3.75 \mathrm{~kg}, \mathrm{P}<0.0001)$. There was no significant difference between the insulin + tofogliflozin group and the glargine + tofogliflozin group (Fig. 4).

\section{Changes in systolic blood pressure (SBP) and diastolic blood pressure (DBP)}

The change in SBP from baseline was $1.1 \mathrm{~mm} \mathrm{Hg}$ in the insulin group (95\% CI: -5.83 to $7.97 \mathrm{~mm} \mathrm{Hg}$ ), which was a slight increase. In contrast, the changes in SBP were $-4.2 \mathrm{~mm} \mathrm{Hg}$ in the insulin + tofogliflozin group (95\% CI: -10.66 to $2.33 \mathrm{~mm} \mathrm{Hg}$ ) and $-7.8 \mathrm{~mm} \mathrm{Hg}$ in the glargine + tofogliflozin group (95\% CI: -15.27 to $-0.26 \mathrm{~mm} \mathrm{Hg}$ ) (Fig. 5 and Table 3). The decrease was significant only in the glargine + tofogliflozin group.

The change in DBP from baseline, similar to SBP, was 0.5 $\mathrm{mm} \mathrm{Hg}$ in the insulin group (95\% CI: -5.60 to $6.67 \mathrm{~mm} \mathrm{Hg}$ ), which was a slight increase. In contrast, the changes in DBP were $-5.6 \mathrm{~mm} \mathrm{Hg}$ in the insulin + tofogliflozin group (95\% CI: -13.71 to $2.49 \mathrm{~mm} \mathrm{Hg}$ ) and $-3.3 \mathrm{~mm} \mathrm{Hg}$ in the glargine + tofogliflozin group (95\% CI: -8.68 to $2.06 \mathrm{~mm} \mathrm{Hg}$ ). DBP tended to decrease (Fig. 6 and Table 3 ).

\section{Change in other parameters}

In the glargine + tofogliflozin group, there was a significant decrease in triglycerides and total cholesterol (Table 3). However, there was no trend toward any increase or decrease in the lipid profiles with the addition of tofogliflozin itself.

The eGFR was significantly decreased in the insulin group and the insulin + tofogliflozin group (Table 3).

\section{Safety and tolerability}

Table 4 shows the incidence of hypoglycemia in each group. 







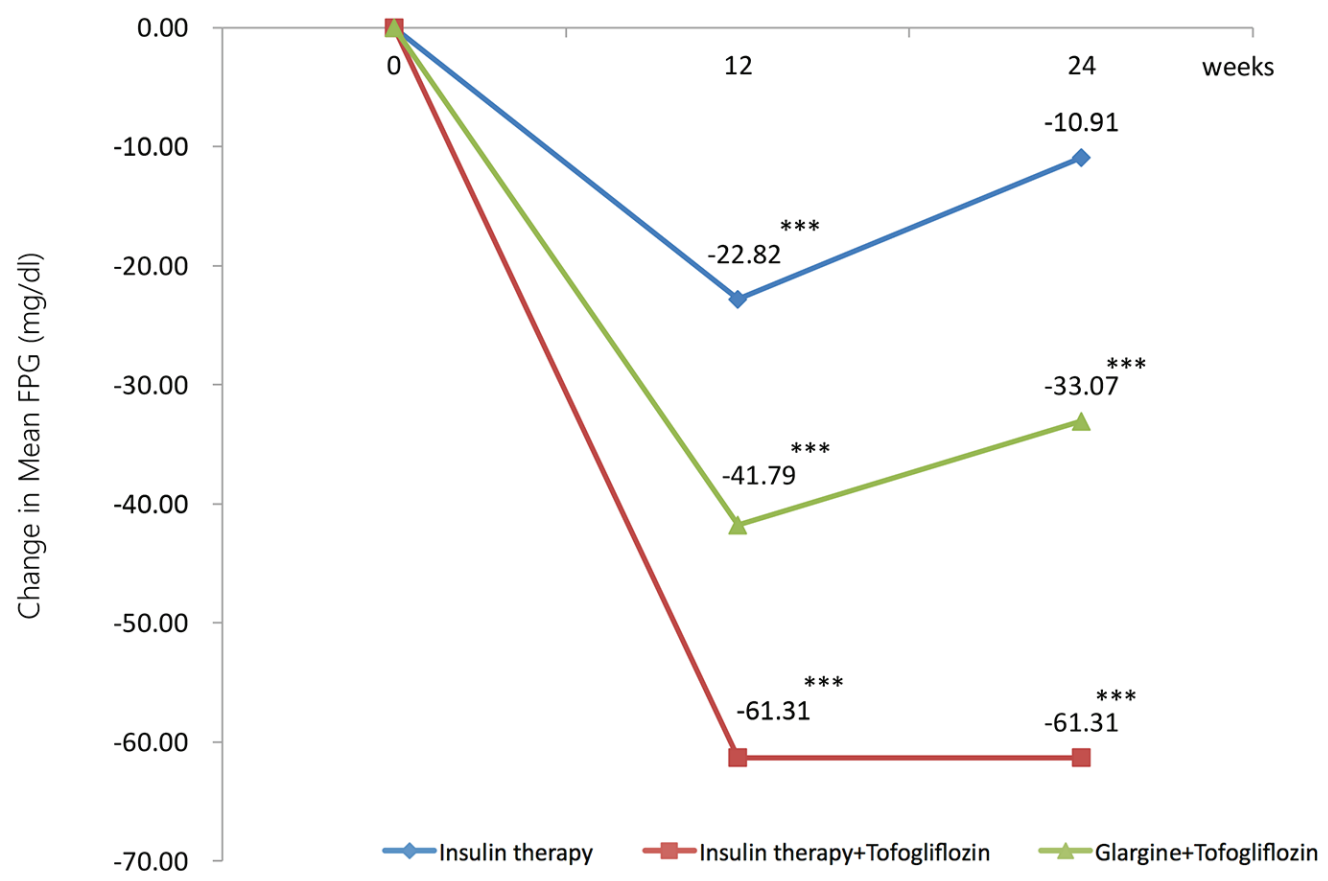

Figure 2. Adjusted mean changes over 24 weeks in fasting plasma glucose (FPG, mg/dL). Data are adjusted mean \pm standard deviation (SD) at baseline and adjusted means $\pm S D$ on treatment. ${ }^{*} P<0.05$ vs. baseline; ${ }^{* *} P<0.01$ vs. baseline; ${ }^{* * *} P<0.001$ vs. baseline by paired t-test.

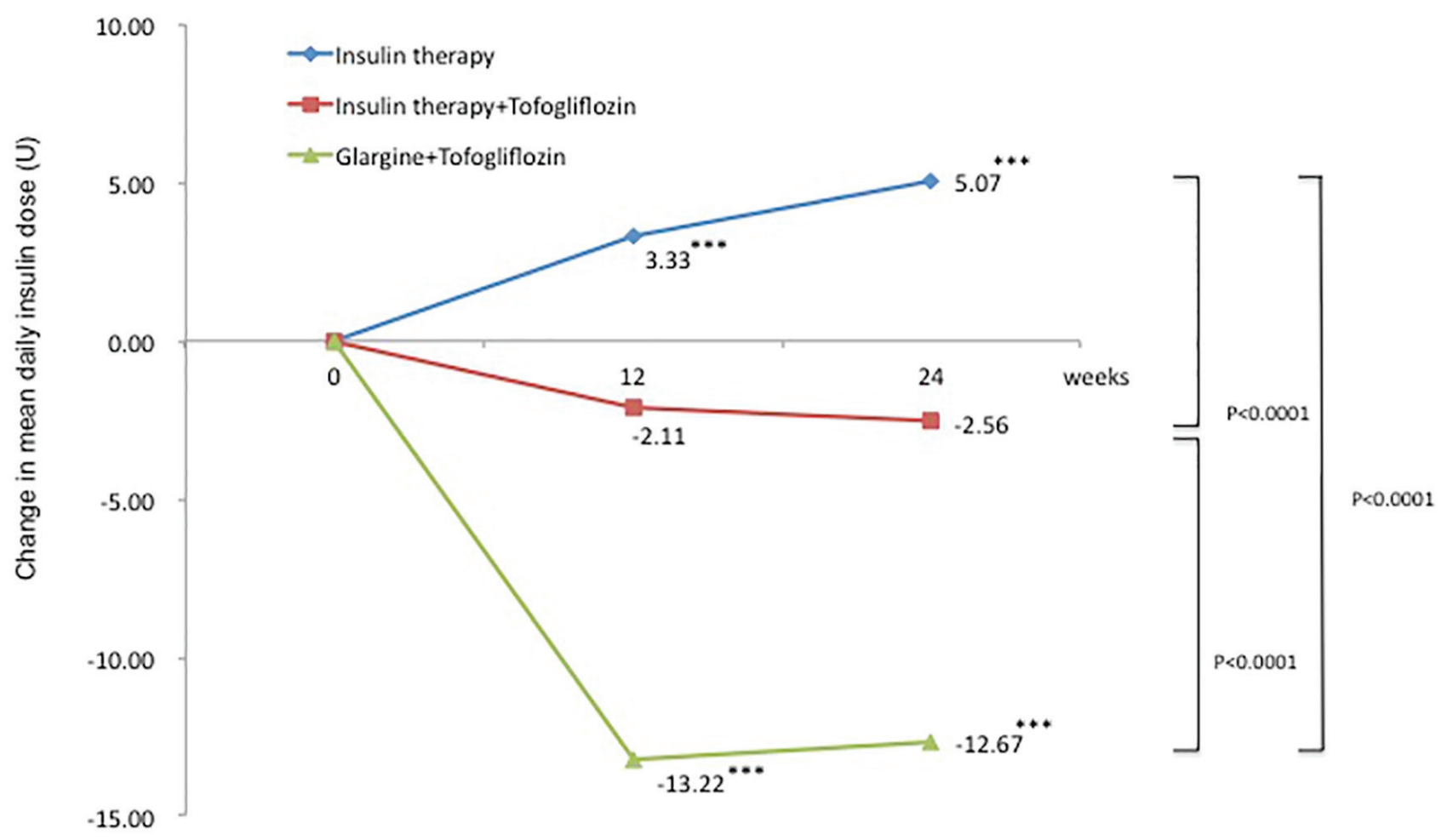

Figure 3. Adjusted mean changes over 24 weeks in total insulin dose (U). Data are adjusted mean \pm standard deviation (SD) at baseline and adjusted mean $\pm \mathrm{SD}$ on treatment. ${ }^{*} \mathrm{P}<0.05 \mathrm{vs}$. baseline; ${ }^{* *} \mathrm{P}<0.01 \mathrm{vs}$. baseline; ${ }^{* * *} \mathrm{P}<0.001 \mathrm{vs}$. baseline by paired t-test. Above $\mathrm{P}$ values were derived using Mann-Whitney analysis to examine between-group differences. 


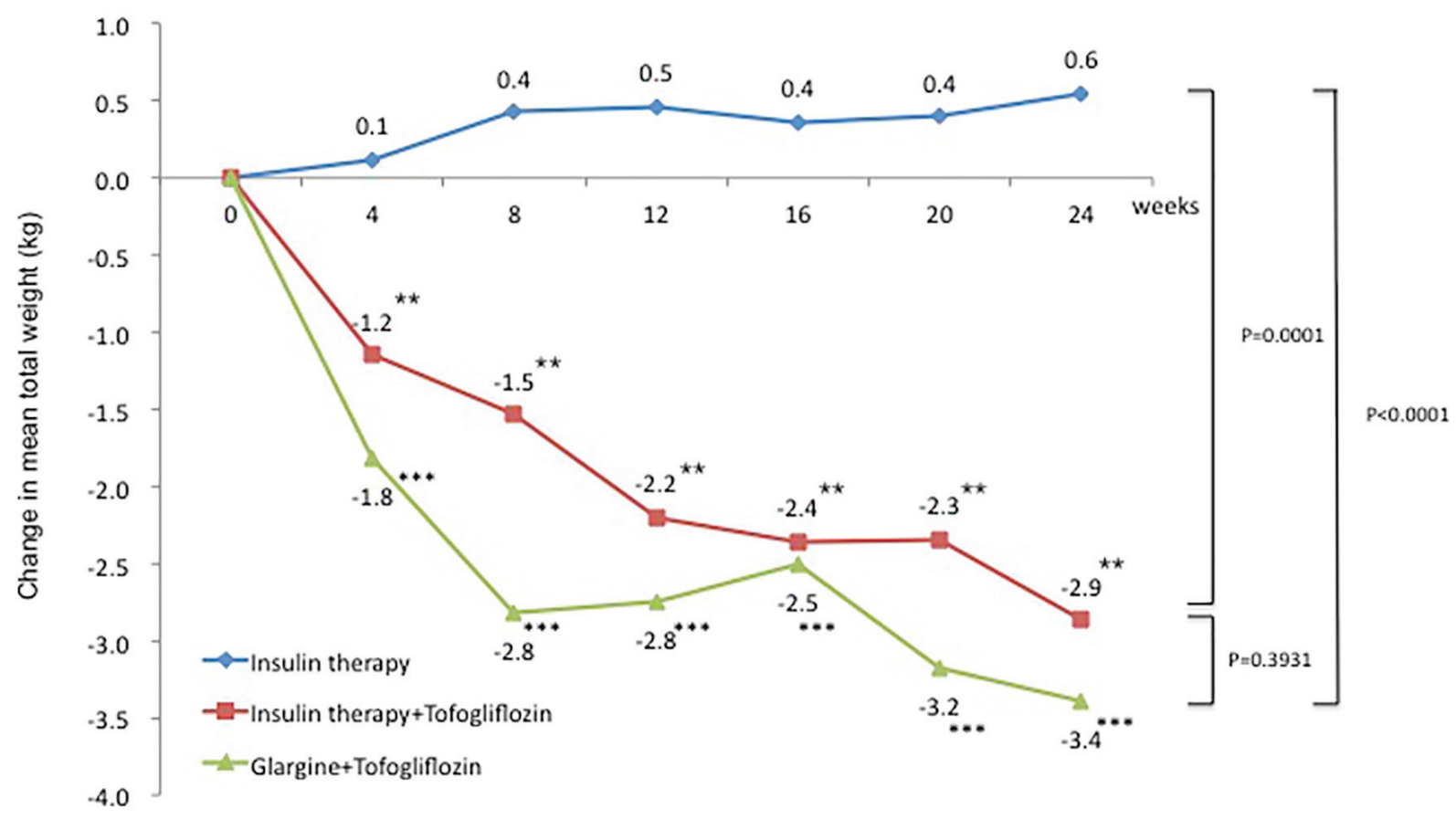

Figure 4. Adjusted mean changes over 24 weeks in weight $(\mathrm{kg})$. Data are adjusted mean \pm standard deviation (SD) at baseline and adjusted mean $\pm S D$ on treatment. ${ }^{*} P<0.05$ vs. baseline; ${ }^{* *} \mathrm{P}<0.01$ vs. baseline; ${ }^{* *} \mathrm{P}<0.001 \mathrm{vs}$. baseline by paired $\mathrm{t}$-test. Above $\mathrm{P}$ values were derived using Mann-Whitney analysis to examine between-group differences.

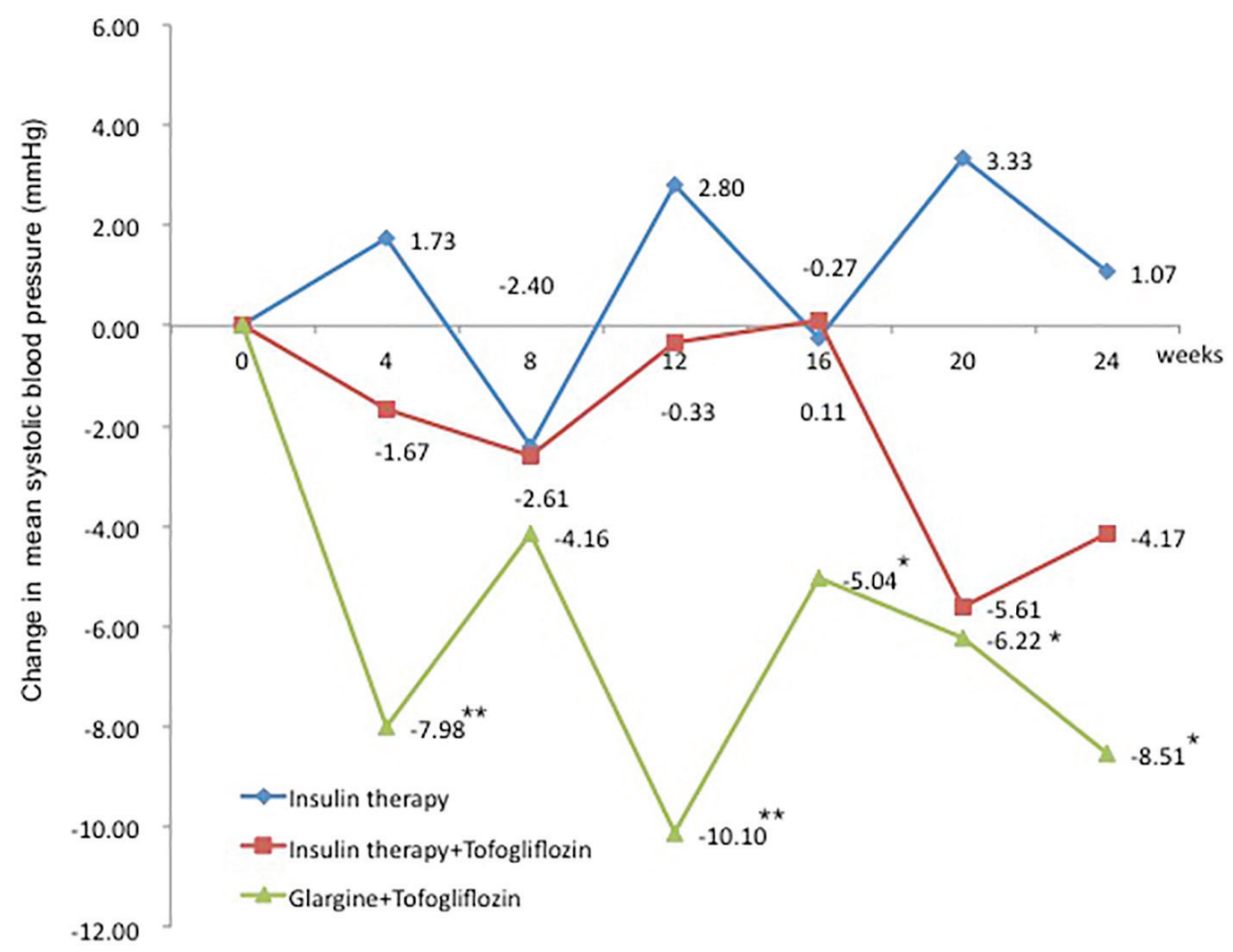

Figure 5. Adjusted mean changes over 24 weeks in systolic blood pressure $(\mathrm{mm} \mathrm{Hg})$. Data are adjusted mean \pm standard deviation (SD) at baseline and adjusted mean $\pm \mathrm{SD}$ on treatment. ${ }^{*} \mathrm{P}<0.05$ vs. baseline; ${ }^{* *} \mathrm{P}<0.01 \mathrm{vs}$. baseline; ${ }^{* * *} \mathrm{P}<0.001 \mathrm{vs}$. baseline by paired t-test analysis. 


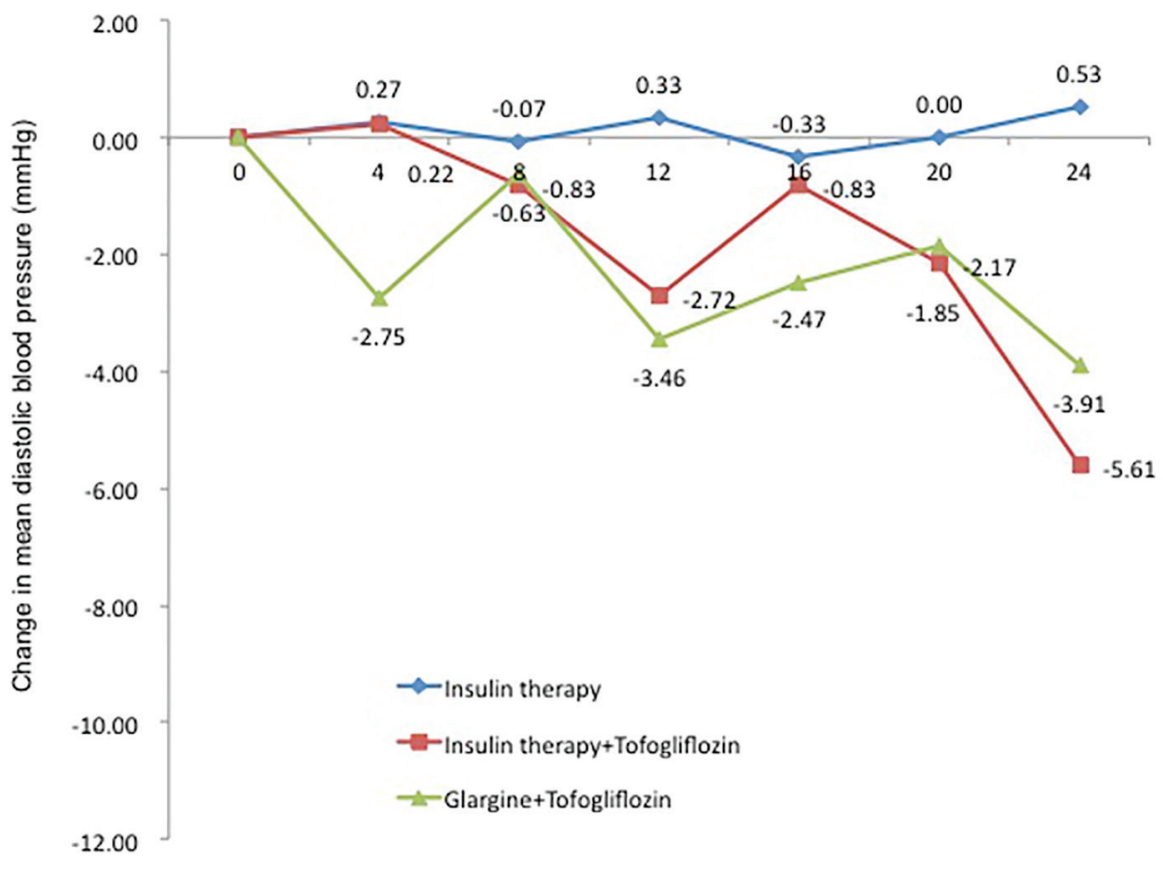

Figure 6. Adjusted mean changes over 24 weeks in diastolic blood pressure ( $\mathrm{mm} \mathrm{Hg}$ ). Data are adjusted mean \pm standard deviation (SD) at baseline and adjusted mean $\pm \mathrm{SD}$ on treatment. ${ }^{*} \mathrm{P}<0.05$ vs. baseline; ${ }^{* *} \mathrm{P}<0.01$ vs. baseline; ${ }^{* *} \mathrm{P}<0.001$ vs. baseline by paired t-test analysis.

Severe hypoglycemia did not occur in any group. Because the occurrence of hypoglycemia was low in each group, the number of patients with hypoglycemia during follow-up is shown. There was no significant difference among the groups.

There were no serious adverse events, and tolerability was excellent.

\section{Discussion}

In T2DM patients on insulin therapy with poor glycemic control, HbAlc in the insulin group initially decreased slightly and then plateaued over the 24-week period. Daily total insulin dose and weight increased during this period, and blood pressure also increased slightly. On the other hand, in the insulin + tofogliflozin group and the glargine + tofogliflozin group, $\mathrm{HbA} 1 \mathrm{c}$ decreased greatly initially, and this continued over the 24-week period, with decreases in $\mathrm{HbA} 1 \mathrm{c}$ of $-1.0 \%$ and $-0.8 \%$, respectively. During this time, total daily insulin dose (-2.6 and $-12.7 \mathrm{U}$, respectively) and weight $(-2.9$ and $-3.4 \mathrm{~kg}$, respectively) decreased, and blood pressure also decreased slightly.

The results of treatment with SGLT2 inhibitors in T2DM patients already on insulin therapy have previously been re- ported overseas. Data from doses similar to standard doses of SGLT2 inhibitors in Japan have been extracted. In a study using canagliflozin $100 \mathrm{mg}$ for 52 weeks [19], significant decreases in $\mathrm{HbA} 1 \mathrm{c}$ of $-0.58 \%$ (95\% CI: -0.68 to $-0.48 \%$ ) and weight of $-2.8 \mathrm{~kg}(95 \% \mathrm{CI}:-7.4$ to $-5.2 \mathrm{~kg})$ compared to a control group were reported. In studies using dapagliflozin 10 $\mathrm{mg}$ for 12 weeks [20] and dapagliflozin $5 \mathrm{mg}$ for 48 weeks [21], significant decreases in HbA1c of $-0.7 \%$ (95\% CI: -1.1 to $-0.3 \%$ ) and $-0.49 \%$ (95\% CI: -0.65 to $-0.33 \%)$, respectively, and significant decreases in weight of $-2.6 \mathrm{~kg}(95 \% \mathrm{CI}:-4.0$ to $-1.2 \mathrm{~kg}$ ) and $-1.82 \mathrm{~kg}(95 \% \mathrm{CI}:-2.56$ to $-1.07 \mathrm{~kg})$, respectively, compared to control groups, were reported.

In other studies using empagliflozin $10 \mathrm{mg}$ for 52 weeks and for 78 weeks $[22,23]$, significant decreases in HbA1c of $-0.44 \%$ (95\% CI: -0.59 to $-0.29 \%)$ and $-0.5 \%$ (95\% CI: -0.7 to $-0.2 \%)$, respectively, and significant decreases in weight of $-2.39 \mathrm{~kg}$ (95\% CI: -3.40 to $-1.39 \mathrm{~kg})$ and $-2.9 \mathrm{~kg}$ (95\% CI: -4.9 to $-1.5 \mathrm{~kg}$ ), respectively, compared to control groups, were reported. No increases in adverse events such as hypoglycemia or ketoacidosis compared to the control groups were reported in any of those studies.

When compared to the present study results (usual dose of tofogliflozin is $20 \mathrm{mg}$ in Japan, corresponding to the pre-

Table 4. Treatment-Emergent Adverse Events

\begin{tabular}{llll} 
& Insulin $(\mathbf{n}=\mathbf{1 5})$ & Insulin + tofogliflozin $(\mathbf{n}=\mathbf{1 9})$ & Glargine + tofogliflozin $(\mathbf{n}=\mathbf{1 9})$ \\
\hline Hypoglycemia $(\mathrm{n})$ & 4 & 5 & 4 \\
\hline
\end{tabular}

*Patient self-reporting only. Number of patients with hypoglycemia during the follow-up period. There were no significant differences among the groups. 
sent insulin + tofogliflozin group), the changes in HbAlc at 24 weeks in the above studies were about the same, namely, a significant decrease of about $-1.0 \%$ in HbAlc. The decreases in weight at 24 weeks were also similar to those in the present study. Moreover, the baseline HbAlc levels in those studies were similar to those in the present patients. Therefore, the effects of SGLT2 inhibitor add-on therapy on HbA1c and weight in T2DM patients already on insulin therapy are probably similar in Western and Japanese patients.

However, in the present patients, the total daily insulin dose decreased $-2.6 \mathrm{U}(-6.2 \%)$ with the addition of tofogliflozin. In the above previous overseas studies, insulin doses increased in the placebo groups, whereas there was no change (no increase or decrease) in the SGLT2 inhibitor add-on therapy groups. The absolute weight reduction was similar in the present patients and the overseas patients. Therefore, because Japanese persons tend to weigh less than Westerners, the same degree of weight loss is likely to correct insulin resistance to a greater extent in Japanese patients. Thus, insulin resistance may be improved to such an extent that the total insulin dose can be decreased.

The use of glargine + tofogliflozin as a novel treatment strategy in the present study is worthy of attention. HbA1c of course improved, but this regimen also had the greatest effects on weight loss and decrease in total insulin dose. Insulin is an anabolic hormone that promotes lipid synthesis. Therefore, patients on insulin therapy with weight gain are often seen in clinical practice. The present glargine + tofogliflozin group had a large decrease in insulin dose from prior therapy based on the protocol (starting with a $30 \%$ total insulin dose reduction from prior treatment). This may have reduced insulin-induced lipid synthesis, thus promoting weight loss. Tofogliflozin has potent effects on correcting postprandial hyperglycemia; thus combined treatment with basal insulin (glargine) only, without the need for bolus insulin, can improve $\mathrm{HbAlc}$. In addition, without the need for bolus insulin, snacking for hypoglycemia may decrease, which also can lead to weight loss.

The present study did not evaluate patient treatment adherence, but Peyrot et al reported increased burden in diabetic patients who used frequent insulin injections [18]. Therefore, with glargine + tofogliflozin, the reduction in total insulin dose together with only one daily insulin injection reduces patient burden, thus presumably contributing to improved glycemic control. Therefore, in T2DM patients poorly controlled on insulin therapy, tofogliflozin + once-daily injection of glargine may be an effective treatment strategy.

The influence of SGLT2 inhibitors on lipid metabolism has been reported previously. Findings such as a rise in HDL cholesterol or fall in triglycerides were not clearly observed in the present study. The reason why no large changes occurred is probably because most $(79.8 \%)$ of the patients in the present study were already using statin drugs and had lipid profiles that were within normal limits. Tofogliflozin add-on therapy was associated with a decrease in eGFR. However, a reduction in eGFR for up to approximately 6 months, followed later by recovery and then maintenance of eGFR has previously been reported [24]. In other words, tofogliflozin does not worsen renal function. Circulating volume is transiently reduced after administration of tofogliflozin, glomerular hyperfiltration is restored to normal filtration, and with an improvement in glucose levels, eGFR may be apparently decreased.

The present study showed the following benefits of tofogliflozin add-on therapy to insulin therapy: 1) tofogliflozin can promote weight loss, which is increased by insulin therapy, thus preventing obesity; 2) tofogliflozin can correct postprandial hyperglycemia not suppressed by an insulin preparation; and 3) tofogliflozin can reduce required insulin doses.

This study has limitations. The relatively small number of patients and the short duration of the follow-up period prevented accurate assessment of the effectiveness of the treatment. Large-scale, long-term, randomized, controlled studies that evaluate endogenous insulin secretion ability are needed to confirm the findings of the present study.

In conclusion, in patients whose T2DM was inadequately controlled despite high-dose insulin therapy, adding tofogliflozin and the combination of tofogliflozin and glargine reduced HbA1c levels, weight, and the dose of insulin over 24 weeks without increasing rates of hypoglycemia. Conversely, in patients who received insulin dose-increase therapy, a progressive increase in insulin dose and weight was observed. Overall, tofogliflozin therapy was well tolerated. These data suggest that tofogliflozin may offer a new treatment option for patients whose T2DM remains inadequately controlled on insulin therapy with or without additional oral glucose-lowering agents.

\section{Acknowledgments}

The authors would like to acknowledge the patients and staff at participating sites for this study. The authors would also like to thank Forte Science Communications (https://www.fortescience.co.jp) for English language review.

\section{Conflicts of Interest}

None of the authors have any potential conflicts of interest associated with this research.

\section{Disclosure}

This study was accepted for a poster presentation at the 59th Annual Meeting of the Japan Diabetes Society, Kyoto, May $19-21,2016$.

\section{References}

1. Hermansen K, Davies M, Derezinski T, Martinez Ravn G, Clauson P, Home P. A 26-week, randomized, parallel, treat-to-target trial comparing insulin detemir with NPH insulin as add-on therapy to oral glucose-lowering drugs in insulin-naive people with type 2 diabetes. Diabetes Care. 2006;29(6):1269-1274.

2. Holman RR, Thorne KI, Farmer AJ, Davies MJ, Keenan JF, Paul S, Levy JC. Addition of biphasic, prandial, or 
basal insulin to oral therapy in type 2 diabetes. $\mathrm{N}$ Engl $\mathrm{J}$ Med. 2007;357(17):1716-1730.

3. Turner RC, Cull CA, Frighi V, Holman RR. Glycemic control with diet, sulfonylurea, metformin, or insulin in patients with type 2 diabetes mellitus: progressive requirement for multiple therapies (UKPDS 49). UK Prospective Diabetes Study (UKPDS) Group. JAMA. 1999;281(21):2005-2012.

4. Bretzel RG, Nuber U, Landgraf W, Owens DR, Bradley C, Linn T. Once-daily basal insulin glargine versus thricedaily prandial insulin lispro in people with type 2 diabetes on oral hypoglycaemic agents (APOLLO): an open randomised controlled trial. Lancet. 2008;371(9618):10731084.

5. Heine RJ, Van Gaal LF, Johns D, Mihm MJ, Widel MH, Brodows RG. Exenatide versus insulin glargine in patients with suboptimally controlled type 2 diabetes: a randomized trial. Ann Intern Med. 2005;143(8):559-569.

6. Nauck MA, Duran S, Kim D, Johns D, Northrup J, Festa A, Brodows R, et al. A comparison of twice-daily exenatide and biphasic insulin aspart in patients with type 2 diabetes who were suboptimally controlled with sulfonylurea and metformin: a non-inferiority study. Diabetologia. 2007;50(2):259-267.

7. Korytkowski M. When oral agents fail: practical barriers to starting insulin. Int J Obes Relat Metab Disord. 2002;26(Suppl. 3):518-524.

8. Guler S, Vaz JA, Ligthelm R. Intensification lessons with modem premixes: from clinical trial to clinical practice. Diabetes Res Clin Pract. 2008;81(Suppl.1):523-530.

9. Wright EM, Hirayama BA, Loo DF. Active sugar transport in health and disease. J Intern Med. 2007;261(1):3243.

10. Rahmoune H, Thompson PW, Ward JM, Smith CD, Hong G, Brown J. Glucose transporters in human renal proximal tubular cells isolated from the urine of patients with noninsulin-dependent diabetes. Diabetes. 2005;54(12):34273434.

11. Defronzo RA. Banting Lecture. From the triumvirate to the ominous octet: a new paradigm for the treatment of type 2 diabetes mellitus. Diabetes. 2009;58(4):773-795.

12. Suzuki M, Honda K, Fukazawa M, Ozawa K, Hagita H, Kawai T, Takeda M, et al. Tofogliflozin, a potent and highly specific sodium/glucose cotransporter 2 inhibitor, improves glycemic control in diabetic rats and mice. J Pharmacol Exp Ther. 2012;341(3):692-701.

13. Tanizawa Y, Kaku K, Araki E, Tobe K, Terauchi Y, Utsunomiya $\mathrm{K}$, Iwamoto $\mathrm{Y}$, et al. Long-term safety and efficacy of tofogliflozin, a selective inhibitor of sodium-glucose cotransporter 2, as monotherapy or in combination with other oral antidiabetic agents in Japanese patients with type 2 diabetes mellitus: multicenter, open-label, randomized controlled trials. Expert Opin Pharmacother.
2014;15(6):749-766.

14. Kaku K, Watada H, Iwamoto Y, Utsunomiya K, Terauchi Y, Tobe K, Tanizawa Y, et al. Efficacy and safety of monotherapy with the novel sodium/glucose cotransporter-2 inhibitor tofogliflozin in Japanese patients with type 2 diabetes mellitus: a combined Phase 2 and 3 randomized, placebo-controlled, double-blind, parallel-group comparative study. Cardiovasc Diabetol. 2014;13:65.

15. Inagaki N. Medical therapy of the diabetes treatment to greatly unfold. How does the SGLT2 inhibitor change diabetes treatment? J New Rem Clin. 2013;62(12):22222231.

16. Buse J. Combining insulin and oral agents. Am J Med. 2000;108(Suppl 6a):23S-32S.

17. Vijan S, Hayward RA, Ronis DL, Hofer TP. Brief report: the burden of diabetes therapy: implications for the design of effective patient-centered treatment regimens. J Gen Intern Med. 2005;20(5):479-482.

18. Peyrot M, Rubin RR, Kruger DF, Travis LB. Correlates of insulin injection omission. Diabetes Care. 2010;33(2):240-245.

19. Neal B, Perkovic V, de Zeeuw D, Mahaffey KW, Fulcher G, Ways K, Desai M, et al. Efficacy and safety of canagliflozin, an inhibitor of sodium-glucose cotransporter 2, when used in conjunction with insulin therapy in patients with type 2 diabetes. Diabetes Care. 2015;38(3):403-411.

20. Wilding JP, Norwood P, T'Joen C, Bastien A, List JF, Fiedorek FT. A study of dapagliflozin in patients with type 2 diabetes receiving high doses of insulin plus insulin sensitizers: applicability of a novel insulin-independent treatment. Diabetes Care. 2009;32(9):1656-1662.

21. Wilding JP, Woo V, Soler NG, Pahor A, Sugg J, Rohwedder K, Parikh S. Long-term efficacy of dapagliflozin in patients with type 2 diabetes mellitus receiving high doses of insulin: a randomized trial. Ann Intern Med. 2012;156(6):405-415.

22. Rosenstock J, Jelaska A, Frappin G, Salsali A, Kim G, Woerle HJ, Broedl UC. Improved glucose control with weight loss, lower insulin doses, and no increased hypoglycemia with empagliflozin added to titrated multiple daily injections of insulin in obese inadequately controlled type 2 diabetes. Diabetes Care. 2014;37(7):18151823.

23. Rosenstock J, Jelaska A, Zeller C, Kim G, Broedl UC, Woerle HJ. Impact of empagliflozin added on to basal insulin in type 2 diabetes inadequately controlled on basal insulin: a 78-week randomized, double-blind, placebocontrolled trial. Diabetes Obes Metab. 2015;17(10):936948.

24. Wanner C, Inzucchi SE, Lachin JM, Fitchett D, von Eynatten M, Mattheus M, Johansen OE, et al. Empagliflozin and Progression of Kidney Disease in Type 2 Diabetes. N Engl J Med. 2016;375(4):323-334. 